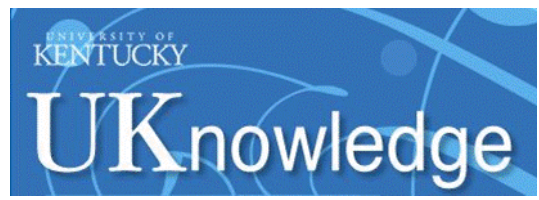

University of Kentucky

UKnowledge

Microbiology, Immunology, and Molecular Genetics Faculty Publications

$5-1-2017$

\title{
Are CDI Systems Multicolored, Facultative, Helping Greenbeards?
}

\author{
Elizabeth S. Danka \\ University of North Carolina at Chapel Hill \\ Erin C. Garcia \\ University of Kentucky, erin.garcia@uky.edu \\ Peggy A. Cotter \\ University of North Carolina at Chapel Hill
}

Follow this and additional works at: https://uknowledge.uky.edu/microbio_facpub

Part of the Bacteria Commons, Cell and Developmental Biology Commons, and the Microbiology

Commons

Right click to open a feedback form in a new tab to let us know how this document benefits you.

\section{Repository Citation}

Danka, Elizabeth S.; Garcia, Erin C.; and Cotter, Peggy A., "Are CDI Systems Multicolored, Facultative, Helping Greenbeards?" (2017). Microbiology, Immunology, and Molecular Genetics Faculty Publications. 138.

https://uknowledge.uky.edu/microbio_facpub/138

This Review is brought to you for free and open access by the Microbiology, Immunology, and Molecular Genetics at UKnowledge. It has been accepted for inclusion in Microbiology, Immunology, and Molecular Genetics Faculty Publications by an authorized administrator of UKnowledge. For more information, please contact UKnowledge@lsv.uky.edu. 


\section{Are CDI Systems Multicolored, Facultative, Helping Greenbeards?}

Digital Object Identifier (DOI)

https://doi.org/10.1016/j.tim.2017.02.008

Notes/Citation Information

Published in Trends in Microbiology, v. 25, issue 5, p. 391-401.

(C) 2017 Elsevier Ltd. All rights reserved.

This manuscript version is made available under the CC-BY-NC-ND 4.0 license

https://creativecommons.org/licenses/by-nc-nd/4.0/.

The document available for download is the author's post-peer-review final draft of the article. 


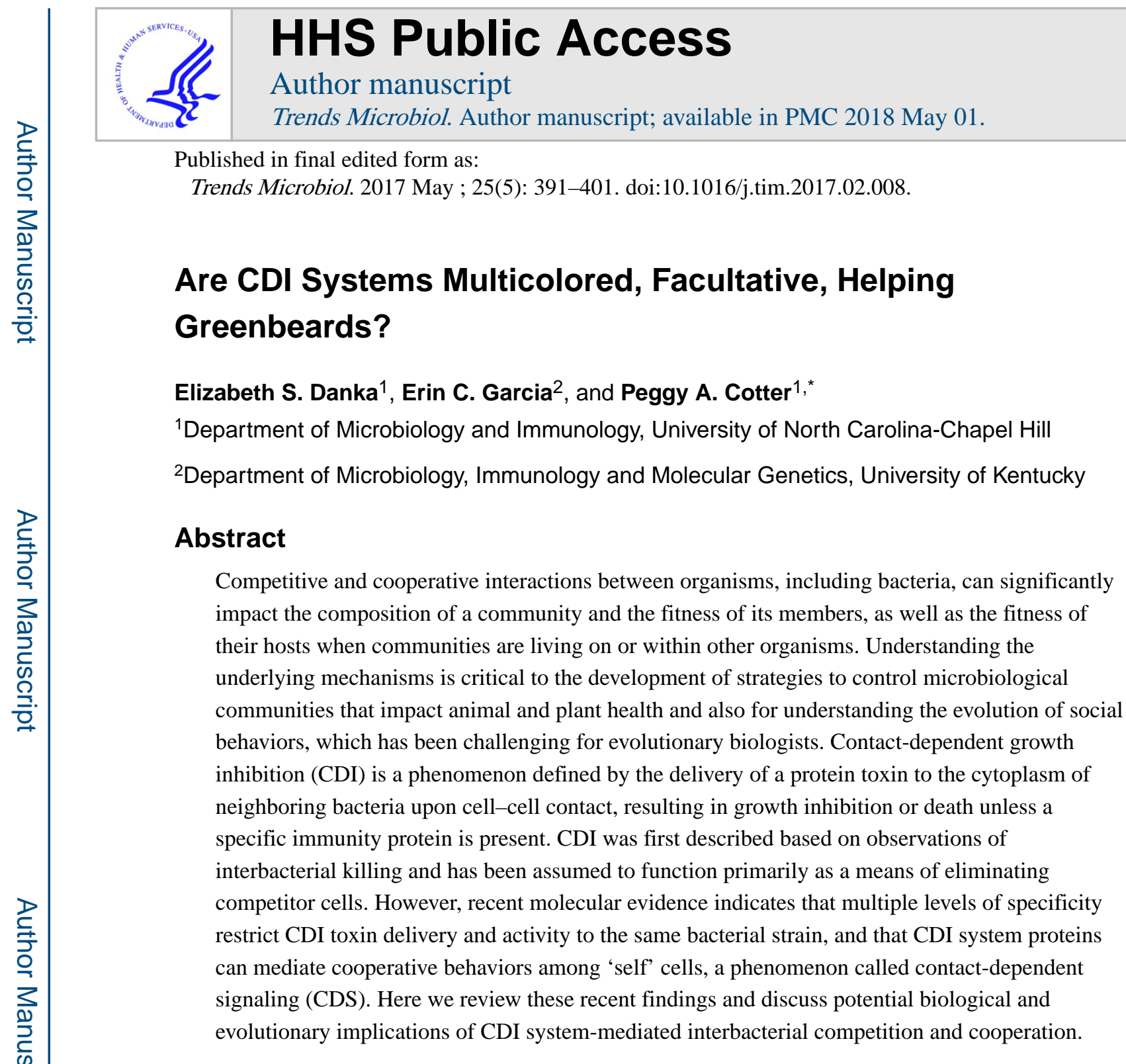

\section{Keywords}

contact-dependent growth inhibition; interbacterial competition; interbacterial cooperation; bacterial signaling; kin/kind selection; greenbeards

\section{Evolution of Sociality}

Explaining cooperation and altruism has been challenging for evolutionary biologists - how can genes encoding behaviors that benefit others at a cost to an individual evolve by natural selection? Hamilton's theory of inclusive fitness (see Glossary) addresses this challenge. Its indirect fitness component states that selection of a gene will be favored if it improves the

\footnotetext{
*Correspondence: peggy_cotter@med.unc.edu (P.A. Cotter).

Publisher's Disclaimer: This is a PDF file of an unedited manuscript that has been accepted for publication. As a service to our customers we are providing this early version of the manuscript. The manuscript will undergo copyediting, typesetting, and review of the resulting proof before it is published in its final citable form. Please note that during the production process errors may be discovered which could affect the content, and all legal disclaimers that apply to the journal pertain.
} 
fitness of other individuals carrying copies of the same gene [1,2]. Key to the evolution of cooperation and altruism, therefore, is identifying and acting upon individuals carrying copies of the same gene. Because genealogical relatives are more likely than non-relatives to share alleles, a gene will increase the probability of persisting in subsequent generations if it improves the fitness of relatives - a process called kin selection. However, if the gene encoding the cooperative or altruistic behavior, or one that is closely linked, encodes a recognizable trait, then individuals carrying the same gene can be identified and targeted directly, irrespective of genealogy - a process called kind selection or 'the greenbeard effect' because of Dawkins' elaboration of a thought experiment put forth by Hamilton. In Dawkins' example, a gene that causes bearers to grow a green beard (or a gene closely linked to the green-beard-encoding gene) causes the bearer to behave nepotistically towards other green-bearded individuals [3]. Greenbeard genes that cause the bearer to cooperate with other individuals bearing the same gene are called helping greenbeards, while those causing the bearer to antagonize individuals that do not bear the same gene are called harming greenbeards (Figure 1) [4,5]. Helping and harming greenbeards can be further distinguished as obligate or facultative, depending on whether they are expressed constitutively or only in response to the presence of greenbeard-bearing (or non-greenbeardbearing) individuals [5].

While a seemingly efficient mechanism for the evolution of cooperation and altruism, greenbeard genes are thought to be rare in nature. One problem is that, if successful, the greenbeard gene will quickly approach fixation, eliminating the diversity necessary for discrimination. Another is that mutant 'falsebeard' cheaters, individuals that reap the benefits of cooperation without paying any of the costs, are likely to arise, which can cause population collapse. Theoretically, these problems can be mitigated by diversity; if multiple allelic variants of the greenbeard genes (multicolored greenbeards) exist in a population, the possibility of one sweeping the population, or of one multifunctional falsebeard taking over, is infinitely small.

Because of their rapid growth, genetic and biochemical tractability, and the relative ease of linking genes to phenotypes, microbes have become important models for investigating molecular mechanisms underlying the evolution of complex social behaviors (e.g., [6-8]). The first identified mechanisms for interbacterial cooperation and competition were those mediated by secreted molecules. Examples include autoinducers that allow bacteria to increase social behaviors in a population density-dependent manner (i.e., quorum sensing) [9], and bacteriocins that intoxicate closely related bacteria that do not produce the correct antitoxin [10]. Indeed, bacteriocins, which are highly polymorphic, have been further investigated as examples of multicolored, obligate, harming greenbeards [4,5,11].

More recent work has described systems that require direct contact between cells for cooperation or competition to occur. Myхососcus xanthus cells in direct end-to-end contact cooperate to form fruiting bodies if the correct surface-bound proteins are present, while excluding cells with incorrect surface proteins [12-14]. Type VI secretion systems (T6SS), found broadly throughout Gram-negative bacterial species, kill neighboring cells by delivering toxic effector proteins [15]. Contact-dependent growth inhibition (CDI) systems also deliver toxic effectors to neighboring cells upon cell-cell contact $[16,17]$. 
While CDI systems were identified based on their ability to mediate competition between neighboring cells, this review summarizes recent data that indicate that these systems can mediate cooperation between siblings, and suggests that they function as multicolored, facultative, helping greenbeards.

\section{Contact-Dependent Growth Inhibition}

$\mathrm{CDI}$ is a phenomenon that was discovered based on the observation that a specific rat fecal isolate of Escherichia coli (strain EC93) inhibited the growth of E. coli K-12 in response to cell-cell contact [16]. The proteins mediating CDI are a subset of two-partner secretion (TPS) pathway proteins that are now known to be widespread among Gram-negative bacteria [17]. CDI systems comprise two major classes: E. coli-type, encoded by $c d i B A I$ loci, which are present in many genera of $\alpha-, \beta$-, and $\gamma$-proteobacteria, and Burkholderiatype, encoded by $b c p A I O B$ loci, which are present in Burkholderia and a few closely related genera (Figure 2A) [17]. As TpsB family members, $\mathrm{CdiB}$ and $\mathrm{BcpB}$ are predicted to be outer membrane $\beta$-barrel proteins that translocate their cognate TpsA family exoproteins, CdiA and BcpA, respectively, across the outer membrane. Typical of TpsA exoproteins, CdiA and BcpA are large (most are $>3000 \mathrm{aa}$ ), contain TPS domains at their N-termini, and are predicted to fold predominantly into $\beta$-helical, rod-shaped structures with their C-termini extending distally from the cell surface. The hallmark of the CDI class of TPS proteins, the $\mathrm{N}$-terminal 2800 aa of CdiA and BcpA proteins are similar, especially among those encoded by the same species, while the C-terminal $~ 300$ aa (termed the CdiA-CT or BcpA$\mathrm{CT}$ ) are highly variable (Figure $2 \mathrm{~B})$. A VENN or Nx(E/Q)LYN motif delineates the conserved and variable regions. The variable CdiA-CT and BcpA-CT domains contain the toxic activity, with most being predicted, and some shown, to function as DNases or RNases (Box 1) [18-23]. When produced within, or delivered to, the cytoplasm of a susceptible bacterium, these toxins degrade DNA or specific tRNAs, resulting in growth inhibition or cell death (Figure 2C). Sibling bacteria are protected from toxicity by producing a small immunity protein, CdiI or BcpI, that is encoded by a gene located immediately 3 to $c d i A$ or $b c p A$, respectively (Figure 2A). CdiI and BcpI proteins covary with CdiA-CT and BcpA-CT (Figure $2 \mathrm{~B}$ ) and protect by binding to and inactivating cognate (encoded by the same allele) but not heterologous (encoded by a different allele) toxins (Box 1) [17,24,25].

\section{Box 1}

\section{Structure and Function of Toxin and Immunity Proteins}

Although many proteins have been described as $\mathrm{CdiA} / \mathrm{BcpA}$ proteins, the mechanisms by which these proteins cause growth inhibition is often unknown. Characterized toxins from Burkholderia pseudomallei, Escherichia coli, Dickeya dadantii (formerly Erwinia chrysanthemi), Pseudomonas aeruginosa, and Enterobacter cloacae have been described to have activities as ribosomal RNases, transfer RNases, pore-forming toxins, or DNases $[17,21,22,25,45,46]$. This functional variability results from structural differences, and from differences in toxin-immunity interactions. The interface at which a toxin and an immunity protein interact can be composed of electrostatic interactions, hydrogen-bonds, ionic bonds, and/or covalent bonds, and may cover 10-20\% of the total surface area of the toxin or immunity domains $[18,21,22]$. In many cases, the complex formed by the 
toxin and immunity proteins has better thermal stability than either protein on its own $[18,21]$. Regardless of toxin mechanism, immunity proteins may alter toxin activity by (i) blocking the active site of the toxin, or (ii) by binding elsewhere and, presumably, changing the structure and/or activity of the toxin. An exposed $\beta$-hairpin from the E. coli EC869 CdiA-CT can stabilize the interaction with CdiI through $\beta$-augmentation [20,47]. This interaction occludes the active site of CdiA, preventing the toxic nuclease activity [20,21]. CdiI also blocks the active site of the CdiA-CTs from E. cloacae and D. dadantii [22]. The toxin domains from $E$. cloacae and $D$. dadantii both form structures resembling the rRNase colicin E3, but neither can access the ribosome to cleave 16S RNA when bound to their cognate immunity proteins $[22,45]$. Toxins from B. pseudomallei $\mathrm{E} 479$ and $1026 \mathrm{~b}$ do not share substantial aa sequence similarity, but both toxins form structures that resemble tRNase colicin E5 and have similarly shaped active sites that allow for preferential cleavage of specific tRNAs $[18,21,25]$. Although the toxin structures are similar, the cognate immunity proteins for these toxins are distinct in sequence, structure, and mechanism of inhibition [18]. BcpI from E479 likely uses a positively-charged protrusion to physically block access of tRNAs into the negatively-charged active site, while BcpI from 1026b seems to bind to an area near the active site, which results in a conformational change that alters the activity of the toxin [18]. Binding of the cognate immunity protein from E. coli EC869 to the toxin domain also results in a change in activity, although a conformational change has not been shown. When in the presence of $\mathrm{Zn}^{2+}$, binding of the immunity protein changes the toxin from a DNase that can degrade supercoiled plasmid DNA and genomic DNA to a DNA nickase [21].

Discovery of the polymorphic nature and covariance of CdiA-CT/BcpA-CT and CdiI/BcpI proteins suggested that $\mathrm{CdiA} / \mathrm{BcpA}$ proteins are modular and that these systems could be used to distinguish 'self' from 'non-self' neighbors, with self being defined by the CdiA-CT/ CdiI or BcpA-CT/BcpI pairs produced by a particular strain [17,24]. In support of this hypothesis, experiments with E. coli and Burkholderia thailandensis showed that, in some cases, grafting heterologous $\mathrm{CT}$ domains onto the constant regions of CdiA or BcpA resulted in functional proteins that can mediate $\mathrm{CDI}$ with the activity and specificity of the protein from which the CT was derived. For example, when overproduced in E. coli, chimeric CdiA proteins that contain the N-terminus from E. coli 536 and the CdiA-CT from E. coli EC93, Dickeya dadantii 3937, or Yersinia pestis CO92, are capable of inhibiting the growth of E. coli K-12 (which lacks cdiBAI genes), while growth inhibition is prevented if the 'target' bacteria produce CdiI from EC93, 3937, or CO92, respectively [17]. Similarly, B. thailandensis E264 producing chimeric BcpA proteins that contain the BcpA-CT encoded by one of two different alleles from Burkholderia pseudomallei 1106a are capable of outcompeting $\triangle b c p A I O B B$. thailandensis E264 that produces no BcpI (or a heterologous $\mathrm{BcpI})$, but not $\triangle b c p A I O B$ B. thailandensis E264 producing cognate BcpI [24]. These studies demonstrate that CDI systems can mediate contact-dependent interbacterial competition. However, the contrived nature of these experiments (e.g., cdiBAIgenes overexpressed from multicopy plasmids and targeting bacteria with mutations in genes encoding surface proteins or in $c d i B A I$ or $b c p A O I B$ ) raises the question of whether CDI systems actually do mediate interbacterial competition in nature, especially for species such as $B$. thailandensis and $B$. 
pseudomallei in which all strains contain at least one $b c p A I O B$ locus (see Outstanding Questions).

\section{Outstanding Questions}

What is the composition of CDS complexes and how do they function mechanistically?

What are the regulatory pathways connecting CDS complexes to gene expression changes?

Do all CDI systems also function as CDS systems?

Are $b c p A I O B / c d i B A I$-containing islands currently mobile and, if so, is mobility stochastically regulated?

Are genes required for CDI and/or CDS also located on $b c p A I O B / c d i B A I-$ containing islands, and, if so, do they act in an allele-specific manner?

Is stochastic expression of $b c p A I O B$ a bet-hedging strategy?

To investigate the biological relevance of CDI, we used B. thailandensis strains that are isogenic except for the DNA region encoding BcpA-CT and BcpI [26]. Although this system is still admittedly artificial, this approach avoids complicating influences by other genes that may contribute to interbacterial competition, such as those encoding T6SS, that differ between strains. These experiments showed that when strains producing different BcpA-CT and $\mathrm{BcpI}$ proteins interact, the strain that is numerically dominant outcompetes the minority strain, even if that strain is outcompeted when the two strains are mixed at a 1:1 ratio. Moreover, strains producing different BcpA-CT and BcpI proteins segregate during biofilm formation, and a biofilm formed by one strain can prevent bacteria producing a different BcpA-CT and BcpI from joining the biofilm. These results support the hypothesis that CDI systems can be used to distinguish self from non-self and to competitively exclude non-self bacteria from a microbial community. In this context, CDI-encoding genes function as multicolored harming greenbeards - they allow their bearer to identify and decrease the fitness of individuals that do not bear the same allele.

\section{Receptor and Translocator Proteins for CdiA/BcpA Delivery}

Although some chimeric CdiA and BcpA proteins are able to mediate CDI, others are not. For example, CdiA from E. coli 536 containing BcpA-CT from B. pseudomallei 1026b does not mediate CDI against E. coli K-12 [17], and B. thailandensis E264 producing BcpA containing the BcpA-CT from B. pseudomallei 1106a bcpAIOB locus 3 is not capable of outcompeting $\triangle b c p A I O B B$. thailandensis E264 [26]. Moreover, interspecies CDI has not been reported, even between strains in which the CdiA-CT of the inhibitor strain is derived from the target strain [16,17]. Studies from the Hayes and Low groups have revealed the mechanistic basis for at least some of the observed CDI system specificity. Mutagenesis studies identified the outer membrane biogenesis protein BamA as a receptor for CdiA of $E$. coli EC93 [27], and Ruhe et al. showed that it is surface-exposed loops 6 and 7 of BamA, which vary between species, that confer specificity (Figure 3) [28]. However, BamA is not 
the receptor for CdiA of E. coli 536. Instead, this CdiA protein binds a hetero-oligomeric complex of the $\mathrm{OmpF}$ and $\mathrm{OmpC}$ osmoporins, with surface-exposed loops 4 and 5 of OmpC being especially important for the interaction [29]. Although the receptor-binding domain of CdiA is unknown, there is evidence that the region between amino acid 1300 and 1600 is involved (Figure 3) [30]. Comparison of all predicted E. coli CdiA proteins indicates that there are at least four distinct classes based on the amino acid sequences in this region, suggesting that at least two additional receptor specificities exist [29].

A second level of specificity occurs during translocation of the toxin domain across the inner membrane (Figure 3). Sequence and predicted structure comparisons (supported, in some cases, by X-ray crystallography data) indicate that CdiA-CT and BcpA-CT polypeptides comprise two distinct subdomains [24,31]. The $\mathrm{C}$-terminal (C, for catalytic) subdomain contains the toxic activity and is sufficient to bind the cognate immunity protein $[21,25,32]$. The N-terminal ( $\mathrm{T}$, for translocation) subdomain, which is sometimes shared between CdiA$\mathrm{CT}$ or BcpA-CT proteins that contain different $\mathrm{C}$ domains, mediates translocation across the cytoplasmic membrane (Figure 3) [31]. Although direct interactions have not been demonstrated, the $\mathrm{T}$ domain appears to bind a specific integral cytoplasmic membrane protein, often an $\mathrm{ABC}$ transporter membrane permease (Figure 3) [31]. Willett et al. hypothesize that the role of this binding is simply to bring the $\mathrm{C}$ domain into close proximity to the cytoplasmic membrane such that the proton motive force can be harnessed for translocation into the cytoplasm [31]. Each T domain that has been studied so far uses a distinct cytoplasmic membrane protein.

A third level of specificity was identified for CdiA of E. coli 536. Diner et al. discovered that the CdiA-CT of this protein (CdiA-CT ${ }^{\mathrm{EC} 536}$ ) is a latent tRNase that is catalytically active only when bound to the biosynthetic enzyme CysK, an $O$-acetylserine sulfhydrylase [32]. Kaundal et al. found that CysK not only binds to CdiA-CT ${ }^{\mathrm{EC} 536}$, it also increases the affinity of CdiA-CT ${ }^{\mathrm{EC} 536}$ for its cognate CdiI protein, stabilizing the CdiA-CT/CdiI complex [23]. The authors propose that in addition to activating the CdiA-CT ${ }^{\mathrm{EC} 536}$ toxin in susceptible cells, CysK may play a crucial role in preventing autoinhibition between neighboring siblings.

Together, these data indicate that self/non-self recognition by CDI systems requires more than just the identity of the CdiA-CT/CdiI or BcpA-CT/BcpI pair produced by a particular bacterium; delivery of the $\mathrm{CT}$ into a neighboring cell requires specific outer and inner membrane proteins and, in at least one case, the toxic activity of the $\mathrm{C}$ domain requires an accessory factor. For those that have been studied, strains producing a specific CDI system are susceptible to that system if their immunity-encoding gene has been deleted, but strains containing different alleles are not always susceptible [17,25,29]. While competition may be fiercest between the most closely related organisms (who are competing for the same resources in the same niches), these observations suggest that, in some cases, the only bacteria that are susceptible to a specific CDI system are those that are so closely related that they also carry the same allele and hence are immune to the toxic activity of the $\mathrm{C}$ domain. Is interbacterial competition, therefore, really the true function of CDI system proteins? 


\section{Contact-Dependent Cooperation}

Although they were not identified as CDI systems at the time, several CdiA/BcpA proteins were shown to play roles in cell-cell aggregation or biofilm formation [33-36]. Several nonCDI TPS pathway proteins also mediate biofilm formation. In Bordetella pertussis, for example, interbacterial aggregation and biofilm formation result from homotypic interactions between the $\beta$-helical shaft of filamentous hemagglutinin (FHA; a TpsA protein) on neighboring bacteria [37,38]. In E. coli strain EC93, CdiA similarly facilitates biofilm development by functioning as an interbacterial adhesin, both through interactions with BamA and through receptor-independent interactions, possibly via CdiA-CdiA adhesion [30]. As aggregation and biofilm formation are considered beneficial behaviors (they provide protection from both biotic and abiotic stress) [39], CDI system-encoding genes, in this context, may function as helping greenbeards, although the extent to which these interactions are allele-specific is unclear.

By contrast, biofilm formation in $B$. thailandensis requires the catalytic activity of BcpA [40]. Other phenotypes, such as production of a pigment and a Congo Red-binding surface structure, also require BcpA catalytic activity, but interbacterial killing is not required, as constitutive expression of $b c p I$ does not abrogate these phenotypes [40]. These findings suggested the hypothesis that BcpA activity mediates cell-cell signaling between sibling bacteria that exchange BcpA-CT polypeptides, but are immune to the molecule's toxicity because they produce BcpI. RNA-sequencing analysis established that gene expression changes occur in $B$. thailandensis in a BcpA-dependent manner, and lac $Z$ reporter experiments showed that these changes require acquisition of a catalytically active BcpA-CT molecule from neighboring bacteria [41]. Several genes induced by this pathway, which we named contact-dependent signaling (CDS), are required for biofilm formation. Together, these data suggest that CDI system-encoding genes in B. thailandensis (and perhaps other bacteria) function as both helping and harming multicolored greenbeards; they increase the fitness of bacteria that bear the same allele by inducing gene expression changes that lead to beneficial behaviors such as biofilm formation, and they decrease the fitness of (kill) bacteria that do not bear the same allele (if they have the correct receptor and translocation proteins in their membranes).

\section{CDS Requires More Than Just BcpA-CT and Bcpl}

The molecular mechanism of CDS remains to be determined, but several possibilities exist (Figure 4). We assume that delivered BcpA-CT forms a complex with BcpI, as bacteria undergoing CDS are protected from growth inhibition in a BcpI-dependent manner. Other accessory proteins may also be required, similar to the interaction of CysK with CdiA-CT/ $\mathrm{Cdil}^{\mathrm{EC} 536}$. In the case of $B$. thailandensis E264, BcpA-CT, on its own, has DNA nickase (i.e., phosphodiesterase) activity, and a BcpA mutant with two amino acid substitutions in the predicted active site within the $\mathrm{C}$ subdomain has neither DNA nickase activity nor CDS activity [41]. In the mutant BcpA protein, these substitutions may have abolished DNA binding or cleavage activity or, less likely, proper folding of the $\mathrm{C}$ domain (the mutant BcpA-CT can still bind BcpI). We hypothesize, therefore, that the BcpA-CT/BcpI signaling complex also has phosphodiesterase activity, but with altered substrate specificity or 
kinetics. One possibility is that equilibrium dynamics between BcpI-bound and -unbound toxin allows for limited, nonlethal DNase activity (Figure 4A), generating a transcriptional response. Another is that the complex acts on a small nucleotide second messenger, such as c-di-GMP or cAMP (Figure 4D), or a small signaling RNA or RNA/DNA hybrid (Figure 4C). It is also possible that the BcpA-CT/BcpI complex alters transcription by binding DNA or even a protein (in a manner requiring the specific amino acids that are mutated in the catalytically inactive BcpA mutant) (Figure 4B).

Regardless of the mechanism, it appears that the requirements for CDS are more stringent than for CDI. B. thailandensis E264 producing chimeric BcpA proteins that contain a heterologous BcpA-CT and corresponding BcpI are able to intoxicate neighboring ( $\triangle b c p A I O B$ E264) cells via CDI, but cannot signal to neighboring siblings via CDS [26]. Thus, although lack of cognate BcpI results in non-self antagonism, the presence of cognate $\mathrm{BcpI}$ is not sufficient to dictate the self cooperative response. CDS appears to require not just any BcpA-CT/BcpI complex, but the 'correct' BcpA-CT/BcpI complex - one that performs a function that can be interpreted and acted on such that the appropriate response is generated in that particular cell. Our results suggest that the ability of a cell to respond to the activity of a BcpA-CT/BcpI complex is linked to the specific bcpAIOB allele.

\section{CDI/CDS-Encoding Genes Are on Genomic Islands}

Most, if not all, $c d i B A I$ and $b c p A I O B$ genes are located on predicted genomic islands that are variably present in different strains within a particular species $[42,43]$. For example, $B$. pseudomallei strain 1106a contains three different bcpAIOB-containing islands, and each is also present in at least two other sequenced $B$. pseudomallei strains [24]. The distribution of $c d i B A I$ - and $b c p A I O B$-containing islands among strains suggests that these islands are, or were recently, mobile. Ruhe et al. showed that cloning the cdiBAI genes from EC93 onto an F plasmid that lacks the genes encoding a partitioning system and a toxin-antitoxin system (and is hence destabilized), prevents loss of the plasmid from the population in a CDIdependent manner [42]. Although genomic islands are typically quite stable, these results suggest that CDI systems could function to stabilize mobile genetic elements. However, if $c d i B A I$ - and $b c p A I O B$-containing islands are currently mobile, the cdiBAI or bcpAIOB genes will only provide CDI-dependent stabilization if the strain into which the island has moved produces outer membrane receptors and inner membrane translocator permeases that can be used by the island-encoded CDI system, unless those proteins are also encoded on the island. Similarly, advantages conferred by CDS will only be realized if the strain, or the island, additionally encodes proteins or regulatory RNAs required for CDS. The presence of genes required for allele-specific CDI or CDS on $c d i B A I$ - or $b c p A I O B$-containing islands would lend further support to $c d i B A I$ and $b c p A I O B$ genes functioning as greenbeards, as a hallmark of greenbeards is linkage disequilibrium between the gene(s) encoding the identifiable trait and the gene(s) encoding cooperative or competitive behaviors [4,5].

\section{Model for CDI/CDS}

In $E$. coli EC93, the strain in which CDI was discovered, the $c d i B A I$ genes are expressed constitutively. In all other strains, $c d i B A I$ and $b c p A I O B$ genes appear to be under tight 
regulatory control. Expression of the bcpAIOB genes in B. thailandensis E264 decreases to a low level during CDS, which makes teleological sense as a population of identical bacteria will benefit by engaging in cooperative rather than competitive behaviors. However, a small proportion (about $0.1 \%$ ) of bacteria growing in a biofilm, or even in liquid culture, express the $b c p A I O B$ genes at a high level (Figure 5, Key Figure) [24,40]. These data, together with other results discussed in this review, support a model in which bacteria use their CDI/CDS systems to sense when they are growing as a community of siblings. CDS causes a majority of the bacteria in the population to decrease expression of their $b c p A I O B$ genes and to increase expression of genes required for biofilm formation and potentially other cooperative behaviors (cooperators in Figure 5). Stochastic high-level expression of bcpAIOB in a small proportion of bacteria (sentinels in Figure 5), however, allows some cells to be ready to attack non-self invaders, should they be encountered. Minoia et al. showed that mobilization of the ICEclc genomic island in Pseudomonas knackmussi is controlled by a series of stochastic processes such that only about $1-3 \%$ of cells in a population are able to transfer the island to other cells at any given time [44]. If mobilization of $c d i B A I$ and $b c p A I O B$ containing islands is similarly stochastically regulated, some cells (converters in Figure 5) will be poised to transfer the island to bacteria that lack it. Thus, stochastic regulation of bcp $A I O B$ genes would allow some bacteria to eliminate non-self invaders via CDI, while stochastic regulation of island mobility would allow others to deliver the island to non-self invaders, which would convert them to self (with regard to the specific CDI/CDS allele) and would also assure maintenance of the island should the invading bacteria be capable of eliminating the original population. Stochastic regulation of CDI/CDS-encoding genes and, potentially, genomic island mobilization, may therefore be a bet-hedging strategy that maximizes the probability that $c d i B A I / b c p A I O B$ genes are maintained and propagated, fulfilling Dawkins' selfish gene hypothesis. Further support for bet-hedging is the fact that the number of $c$ diBAII bcpAIOB loci in any strain appears to be small, suggesting that they are costly to maintain.

\section{Concluding Remarks}

Although the theory of inclusive fitness and the concept of greenbeard genes were proposed more than half a century ago, the genetic and molecular mechanisms underlying cooperation and altruism remain incompletely understood. The relatively recent appreciation that microbes are social organisms has led to the development of bacterial models for studying complex group behaviors and theories of social evolution. Bacteriocins, especially colicins produced by E. coli, are excellent models for investigating multicolored, obligate, harming greenbeards. The data summarized in this review suggest that CDI/CDS systems may serve as equally useful models for investigating multicolored, facultative, helping greenbeards, leading to substantial advances in our understanding of social evolution. Moreover, it is now well established that bacteria growing in biofilms can cause or exacerbate human disease, and that microbial community composition can affect immune system development and other aspects of human health. Understanding sociomicrobiological behavior therefore also has important public health ramifications. 


\section{Acknowledgments}

We thank members of our laboratory for many useful discussions and for reading multiple versions of the manuscript. We are especially grateful to Ashleigh Griffin who read the manuscript critically and offered helpful advice on the evolutionary biology aspects of the manuscript. Research by the authors' relating to work described in this review was supported by the National Institutes of Health (R21 AI112764 and R01 GM121110 to P.A.C.; F32 AI096728 and K22 AI118949 to E.C.G.; E.S.D. was supported by K12GM00678 from the Training, Workforce Development, and Diversity division of the NIGMS).

\section{Glossary}

\section{Contact-dependent growth inhibition (CDI)}

CdiBAI- or BcpAIOB-dependent killing or growth inhibition via the exchange of toxins between cells that are in direct contact with each other.

\section{Contact-dependent signaling (CDS)}

changes in gene expression triggered by BcpA-CT (or CdiA-CT) toxin exchange between closely related cells containing an immunity protein (BcpI or CdiI) specific to the exchanged toxin.

\section{Direct fitness}

natural selection of a gene that increases the frequency of copies of itself in future generations by improving the fitness of its bearer.

\section{Genomic island}

a contiguous set of genes on a chromosome that has evidence of having been acquired by horizontal transfer (i.e., insertion near tRNA-encoding genes, altered GC content, presence of phage/plasmid elements, and/or flanking repeat sequences).

\section{Greenbeard genes}

genes that cause a phenotypic effect (such as the presence of a green beard or some other conspicuous feature), allow the bearer to recognize this feature in other individuals, and cause the bearer to behave differently towards other individuals depending on whether they display the feature.

\section{Indirect fitness}

natural selection of a gene that increases the frequency of copies of itself in future generations by improving the fitness of other individuals who carry copies of the same gene.

\section{Kin selection}

natural selection of genes encoding behaviors that decrease the fitness of the bearer but increase the fitness of their kin (who share a proportion of their genes).

\section{Kind selection (the greenbeard effect)}

natural selection of genes encoding behaviors that decrease the fitness of the bearer but increase the fitness of individuals that carry copies of the same gene.

\section{Linkage disequilibrium}

nonrandom assortment of genes, such that certain genes are found together at a higher frequency than would be expected if the genes assorted randomly and independently. 


\section{Inclusive fitness}

the sum of direct and indirect fitness.

\section{Selfish gene hypothesis}

adaptive evolution occurs though differential survival of competing genes, increasing the frequency of alleles encoding phenotypic traits that promote their own propagation.

\section{References}

1. Hamilton WD. The genetical evolution of social behaviour. I J Theor Biol. 1964; 7:1-16. [PubMed: 5875341]

2. Hamilton WD. The genetical evolution of social behaviour. II J Theor Biol. 1964; 7:17-52. [PubMed: 5875340]

3. Dawkins, R. The Selfish Gene. Oxford University Press; 1976.

4. West SA, Gardner A. Altruism, spite, and greenbeards. Science. 2010; 327:1341-1344. [PubMed: 20223978]

5. Gardner A, West SA. Greenbeards. Evolution. 2010; 64:25-38. [PubMed: 19780812]

6. Levin BR, Bergstrom CT. Bacteria are different: observations, interpretations, speculations, and opinions about the mechanisms of adaptive evolution in prokaryotes. Proc Natl Acad Sci USA. 2000; 97:6981-6985. [PubMed: 10860960]

7. Levin BR, et al. Compensatory mutations, antibiotic resistance and the population genetics of adaptive evolution in bacteria. Genetics. 2000; 154:985-997. [PubMed: 10757748]

8. Baym M, et al. Spatiotemporal microbial evolution on antibiotic landscapes. Science. 2016; 353:1147-1151. [PubMed: 27609891]

9. Darch SE, et al. Density-dependent fitness benefits in quorum-sensing bacterial populations. Proc Natl Acad Sci USA. 2012; 109:8259-8263. [PubMed: 22566647]

10. Hibbing ME, et al. Bacterial competition: surviving and thriving in the microbial jungle. Nat Rev Microbiol. 2010; 8:15-25. [PubMed: 19946288]

11. Biernaskie JM, et al. Multicoloured greenbeards, bacteriocin diversity and the rock-paper-scissors game. J Evol Biol. 2013; 26:2081-2094. [PubMed: 23980628]

12. Wall D. Molecular recognition in myxobacterial outer membrane exchange: functional, social and evolutionary implications. Mol Microbiol. 2014; 91:209-220. [PubMed: 24261719]

13. Sager B, Kaiser D. Intercellular C-signaling and the traveling waves of Myxococcus. Genes Dev. 1994; 8:2793-2804. [PubMed: 7995518]

14. Jelsbak L, Søgaard-Andersen L. The cell surface-associated intercellular C-signal induces behavioral changes in individual Myхососcus xanthus cells during fruiting body morphogenesis. Proc Natl Acad Sci USA. 1999; 96:5031-5036. [PubMed: 10220413]

15. Russell AB, et al. Type VI secretion system effectors: poisons with a purpose. Nat Rev Microbiol. 2014; 12:137-148. [PubMed: 24384601]

16. Aoki SK, et al. Contact-dependent inhibition of growth in Escherichia coli. Science. 2005; 309:1245-1248. [PubMed: 16109881]

17. Aoki SK, et al. A widespread family of polymorphic contact-dependent toxin delivery systems in bacteria. Nature. 2010; 468:439-442. [PubMed: 21085179]

18. Johnson PM, et al. Functional diversity of cytotoxic tRNase/immunity protein complexes from Burkholderia pseudomallei. J Biol Chem. 2016; 291:19387-19400. [PubMed: 27445337]

19. Johnson PM, et al. Unraveling the essential role of CysK in CDI toxin activation. Proc Natl Acad Sci USA. 2016; 113:9792-9797. [PubMed: 27531961]

20. Morse RP, et al. Diversification of $\beta$-augmentation interactions between CDI toxin/immunity proteins. J Mol Biol. 2015; 427:3766-3784. [PubMed: 26449640]

21. Morse RP, et al. Structural basis of toxicity and immunity in contact-dependent growth inhibition (CDI) systems. Proc Natl Acad Sci USA. 2012; 109:21480-21485. [PubMed: 23236156] 
22. Beck CM, et al. CdiA from Enterobacter cloacae delivers a toxic ribosomal RNase into target bacteria. Structure. 2014; 22:707-718. [PubMed: 24657090]

23. Kaundal S, et al. Dual role of a biosynthetic enzyme, CysK, in contact dependent growth inhibition in bacteria. PLoS ONE. 2016; 11:e0159844. [PubMed: 27458806]

24. Anderson MS, et al. The Burkholderia $b c p A I O B$ genes define unique classes of two-partner secretion and contact dependent growth inhibition systems. PLoS Genet. 2012; 8:e1002877. [PubMed: 22912595]

25. Nikolakakis K, et al. The toxin/immunity network of Burkholderia pseudomallei contact-dependent growth inhibition (CDI) systems. Mol Microbiol. 2012; 84:516-529. [PubMed: 22435733]

26. Anderson MS, et al. Kind discrimination and competitive exclusion mediated by contact-dependent growth inhibition systems shape biofilm community structure. PLoS Pathog. 2014; 10:e1004076. [PubMed: 24743836]

27. Aoki SK, et al. Contact-dependent growth inhibition requires the essential outer membrane protein BamA (YaeT) as the receptor and the inner membrane transport protein AcrB. Mol Microbiol. 2008; 70:323-340. [PubMed: 18761695]

28. Ruhe ZC, et al. Receptor polymorphism restricts contact-dependent growth inhibition to members of the same species. mBio. 2013; 4:e00480-13-e00480-13. [PubMed: 23882017]

29. Beck CM, et al. CdiA Effectors from Uropathogenic Escherichia coli Use Heterotrimeric Osmoporins as Receptors to Recognize Target Bacteria. PLoS Pathog. 2016; 12:e1005925. [PubMed: 27723824]

30. Ruhe ZC, et al. CdiA promotes receptor-independent intercellular adhesion. Mol Microbiol. 2015; 98:175-192. [PubMed: 26135212]

31. Willett JLE, et al. Contact-dependent growth inhibition toxins exploit multiple independent cellentry pathways. Proc Natl Acad Sci USA. 2015; 112:11341-11346. [PubMed: 26305955]

32. Diner EJ, et al. Identification of a target cell permissive factor required for contact-dependent growth inhibition (CDI). Genes Dev. 2012; 26:515-525. [PubMed: 22333533]

33. Rojas CM, et al. HecA, a member of a class of adhesins produced by diverse pathogenic bacteria, contributes to the attachment, aggregation, epidermal cell killing, and virulence phenotypes of Erwinia chrysanthemi EC16 on Nicotiana clevelandii seedlings. Proc Natl Acad Sci USA. 2002; 99:13142-13147. [PubMed: 12271135]

34. Gottig N, et al. A filamentous hemagglutinin-like protein of Xanthomonas axonopodis pv. citri, the phytopathogen responsible for citrus canker, is involved in bacterial virulence. PLoS ONE. 2009; 4:e4358-13. [PubMed: 19194503]

35. Guilhabert MR, Kirkpatrick BC. Identification of Xylella fastidiosa antivirulence genes: hemagglutinin adhesins contribute a biofilm maturation to $X$. fastidios and colonization and attenuate virulence. Mol Plant Microbe Interact. 2005; 18:856-868. [PubMed: 16134898]

36. Neil RB, Apicella MA. Role of HrpA in biofilm formation of Neisseria meningitidis and regulation of the hrpBAS transcripts. Infect Immun. 2009; 77:2285-2293. [PubMed: 19289515]

37. Menozzi FD, et al. Heparin-inhibitable lectin activity of the filamentous hemagglutinin adhesin of Bordetella pertussis. Infect Immun. 1994; 62:769-778. [PubMed: 8112848]

38. Hoffman, C., et al. Bordetella adenylate cyclase toxin interacts with filamentous haemagglutinin to inhibit biofilm formation in vitro. Mol Microbiol. 2016. Published online Month Day, Year. http:// dx.doi.org/10.1111/mmi.13551

39. Hall-Stoodley L, et al. Bacterial biofilms: from the natural environment to infectious diseases. Nat Rev Microbiol. 2004; 2:95-108. [PubMed: 15040259]

40. Garcia EC, et al. Burkholderia BcpA mediates biofilm formation independently of interbacterial contact-dependent growth inhibition. Mol Microbiol. 2013; 89:1213-1225. [PubMed: 23879629]

41. Garcia EC, et al. Interbacterial signaling via Burkholderia contact-dependent growth inhibition system proteins. Proc Natl Acad Sci USA. 2016; 113:8296-8301. [PubMed: 27335458]

42. Ruhe ZC, et al. CDI Systems are stably maintained by a cell-Contact mediated surveillance mechanism. PLoS Genet. 2016; 12:e1006145. [PubMed: 27355474]

43. Tuanyok A, et al. Genomic islands from five strains of Burkholderia pseudomallei. BMC Genomics. 2008; 9:566. [PubMed: 19038032] 
44. Minoia M, et al. Stochasticity and bistability in horizontal transfer control of a genomic island in Pseudomonas. Proc Natl Acad Sci USA. 2008; 105:20792-20797. [PubMed: 19098098]

45. Walker D, et al. Identification of the catalytic motif of the microbial ribosome inactivating cytotoxin colicin E3. Protein Sci. 2004; 13:1603-1611. [PubMed: 15133158]

46. Aoki SK, et al. Contact-dependent growth inhibition causes reversible metabolic downregulation in Escherichia coli. J Bacteriol. 2009; 191:1777-1786. [PubMed: 19124575]

47. Remaut H, Waksman G. Protein-protein interaction through beta-strand addition. Trends Biochem Sci. 2006; 31:436-444. [PubMed: 16828554]

48. Jakes KS, Cramer WA. Border crossings: colicins and transporters. Annu Rev Genet. 2012; 46:209-231. [PubMed: 22934645] 


\section{Trends}

Specificity between $\mathrm{BcpA} / \mathrm{CdiA}$ proteins and receptors and translocator proteins restricts delivery of BcpA-CT/CdiA-CT polypeptides to closely related bacteria.

In Burkholderia thailandensis, delivery of BcpA-CT into an immune cell results in a change in gene expression, a phenomenon called contact-dependent signaling (CDS).

CDI system-encoding genes are located on genomic islands.

CDI system-encoding genes are tightly regulated. In $B$. thailandensis, these genes are expressed stochastically; they are expressed highly in only about $0.1 \%$ of cells growing in culture or in a biofilm.

$b c p A I O B$ genes act as both helping and harming greenbeards; they induce gene expression changes in 'self' bacteria that cause cooperative behaviors and inhibit the growth of, or kill, 'non-self' bacteria.

As multicolored, facultative, helping greenbeards, CDI/CDS systems represent an excellent model for studying social evolution. 


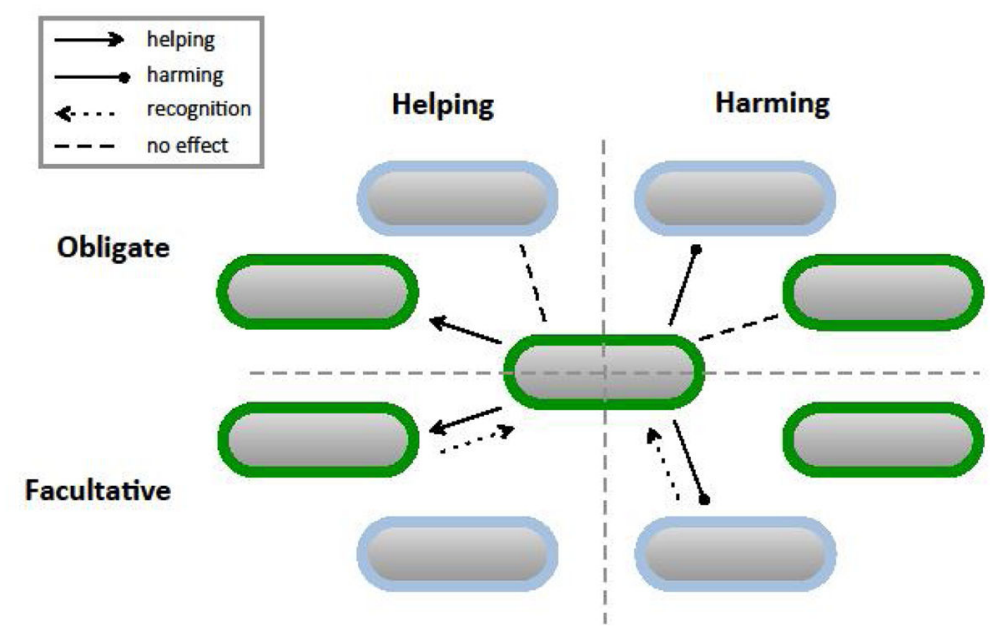

Figure 1. Greenbeard Recognition Leads to Helping or Harming Behaviors

Greenbeards allow cells to recognize related social partners within a population. These loci can be expressed at all times (obligate), or expressed in response to a social partner with or without a greenbeard (facultative). Here, neighboring cells expressing the appropriate greenbeard (cells with green envelopes) will lead to helping behaviors (solid line with arrow), while recognition of neighboring cells without the appropriate greenbeards (cells with blue envelopes) will lead to harming behaviors (solid line with rounded end). Obligate helping behaviors will benefit greenbearded neighbors while neighbors lacking the greenbeard cannot benefit (no effect, dashed line). Obligate harming behaviors will harm neighbors without a greenbeard while greenbearded neighbors are protected (no effect). Facultative helping greenbeards will promote helping behaviors upon recognition (dotted line with arrow) of a greenbearded social partner, while facultative harming greenbeards will result in harming behavior upon recognition of a social partner lacking a greenbeard.

(Adapted from [5].) 


\section{A) CDI system-encoding loci}

E. coli-type

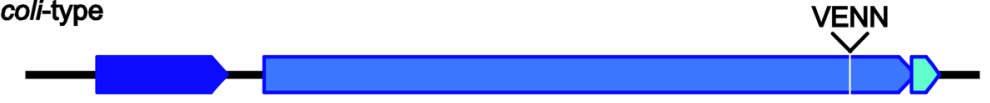

Burkholderia-type

$\mathrm{Nx}(\mathrm{E} / \mathrm{Q}) \mathrm{LYN}$

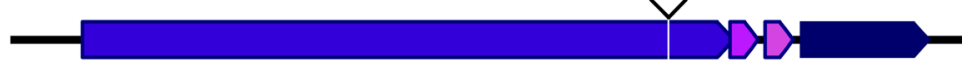

B) Examples of BcpA and Bcpl protein polymorphism

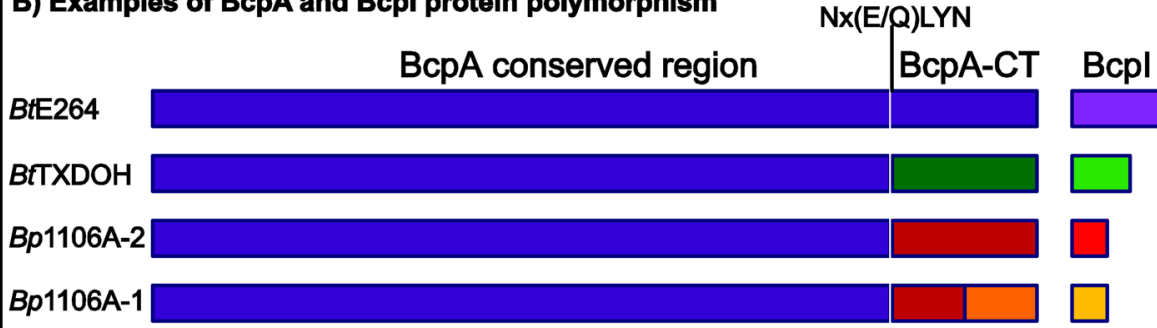

C) Model for CDI

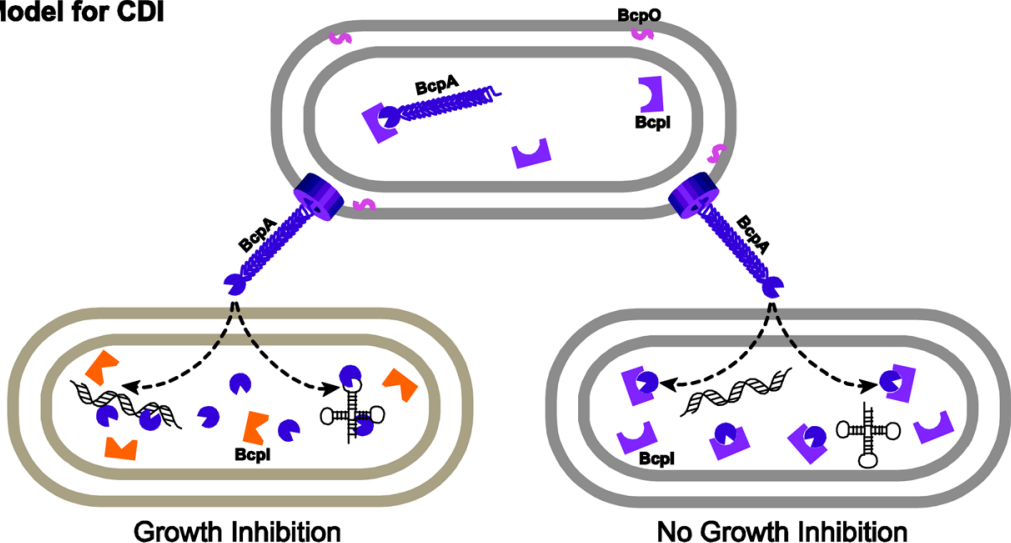

Figure 2. Contact-Dependent Growth Inhibition Systems

(A) Schematic of Escherichia coli- and Burkholderia-type CDI system-encoding loci. The small gene labeled ' $I$ is $c d i I$ or $b c p I$ and the small gene labeled ' $O$ ' is $b c p O$. (B) Four representative $\mathrm{BcpA}$ and $\mathrm{BcpI}$ protein pairs are shown. The different colored BcpA-CT and BcpI proteins indicate $<10 \%$ aa similarity. (C) Model for CDI using Burkholderia thailandensis as an example. The BcpAIOB-producing cell (top) delivers some portion of the BcpA C-terminus to a recipient cell upon cell-cell contact. If the recipient cell produces no BcpI protein, or a BcpI protein encoded by a different allele (gold in the bottom left cell), the toxic C-terminal domain of BcpA will degrade DNA or tRNA, resulting in cell death. If the recipient cell produces BcpI encoded by the same allele as the BcpA-producing cell, $\mathrm{BcpI}$ will bind to the BcpA C-terminus, blocking toxic activity. BcpO (lavender) is a small lipoprotein that is predicted to localize to the inner leaflet of the outer membrane. Its function is unknown. 


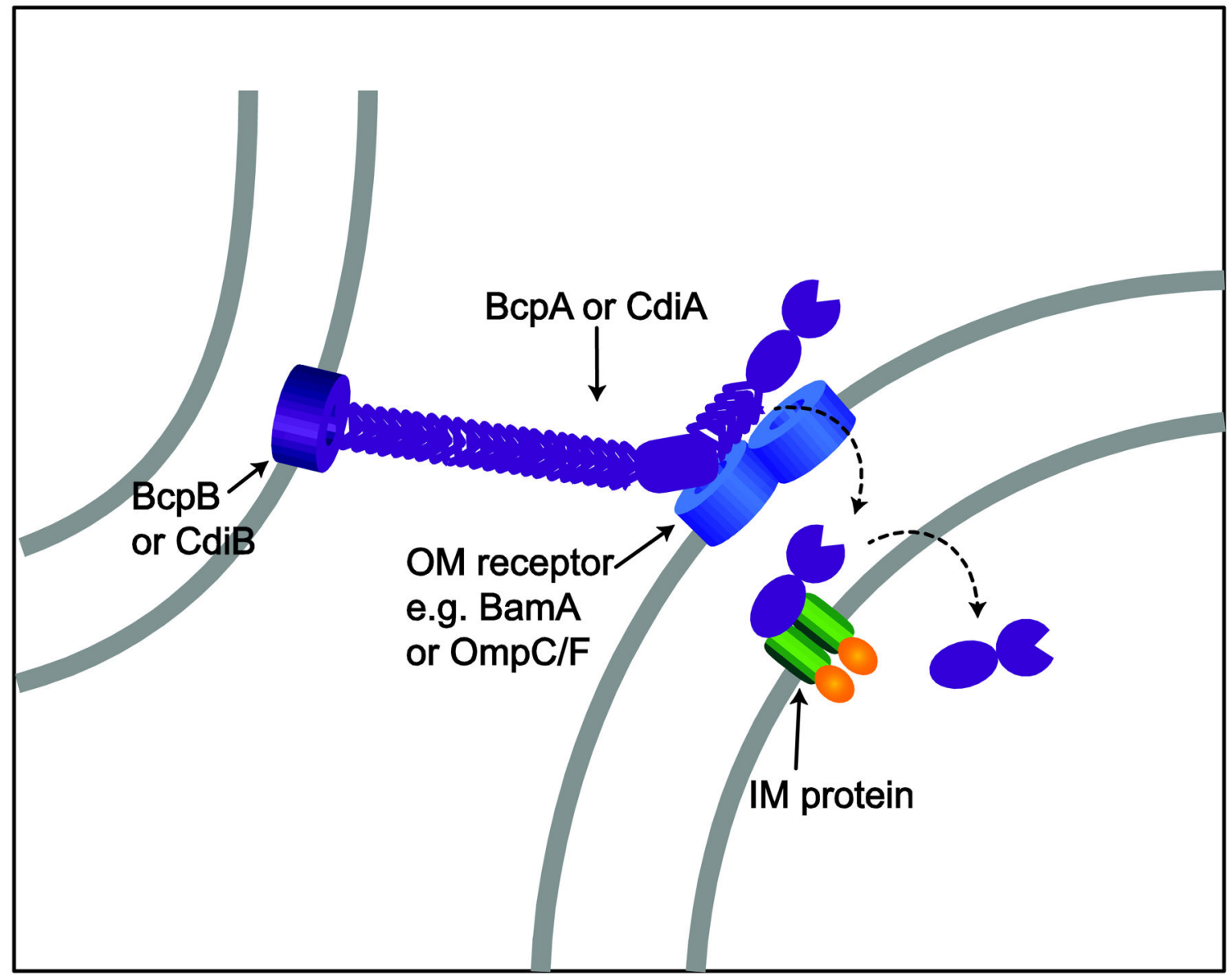

Figure 3. Model for Translocation of BcpA-CT/CdiA-CT into Target Bacteria

Data indicate that a binding (B) domain on BcpA or CdiA must be located within the constant region. Interaction of this domain with an outer membrane receptor (BamA or OmpC/F, in Escherichia coli) somehow triggers translocation of the BcpA-CT/CdiA-CT across the outer membrane (OM) and into the periplasm. Cleavage likely occurs during this step to generate a polypeptide containing the translocation $(\mathrm{T})$ and catalytic $(\mathrm{C})$ domains, but possibly also part of the constant region of $\mathrm{BcpA} / \mathrm{CdiA}$. The $\mathrm{T}$ domain interacts with an inner membrane (IM) protein, and it is hypothesized that the proton-motive force drives translocation of the $\mathrm{T}$ and $\mathrm{C}$ domains into the cytoplasm. This model is based on many elegant studies investigating the mechanism of translocation of bacteriocins ([48] and references therein). 


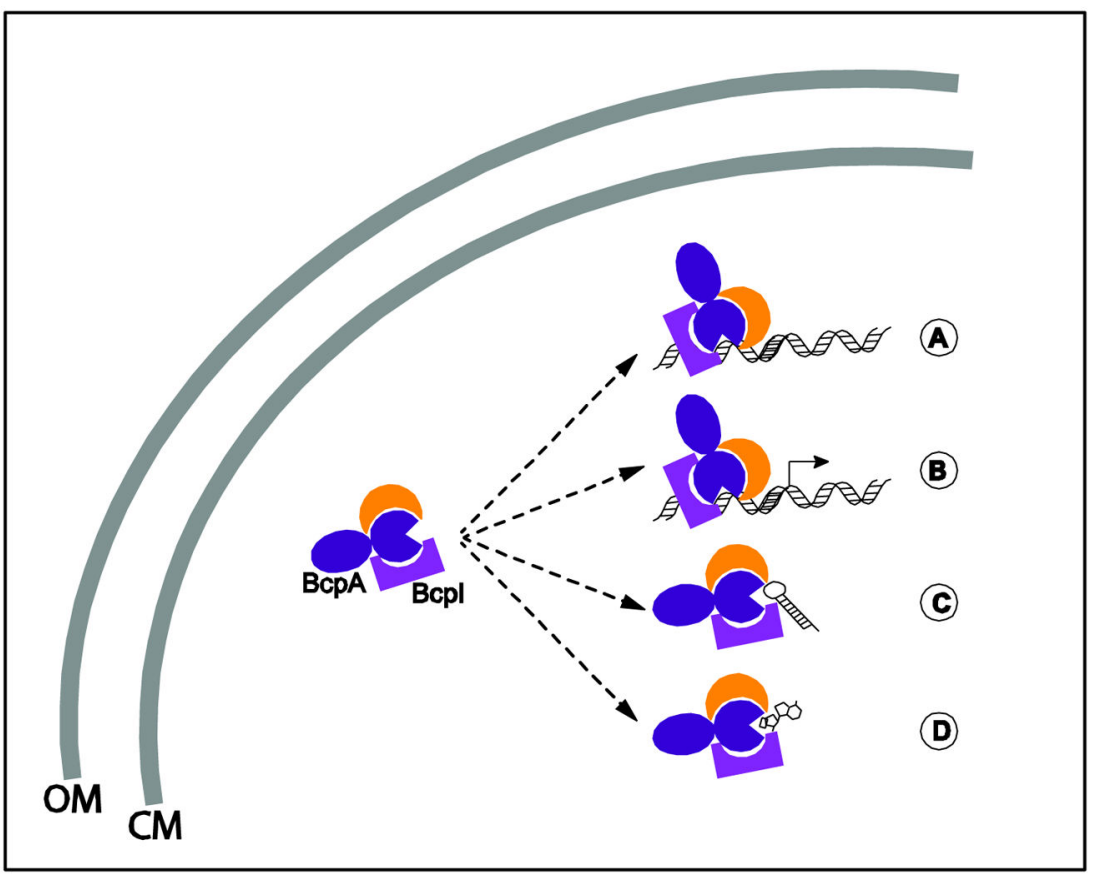

Figure 4. Possible Mechanisms of Contact-Dependent Interbacterial Signaling

An intracellular complex formed by BcpA-CT, BcpI, and possibly other factor(s) (orange crescent) may induce gene expression changes via several potential mechanisms: (A) limited nonlethal DNA nicking, (B) binding to specific DNA sequences to directly activate or repress transcription, (C) interaction with small RNA(s), or (D) interaction with a small nucleotide second messenger. The outer membrane $(\mathrm{OM})$ and cytoplasmic membrane $(\mathrm{CM})$ are labeled. 


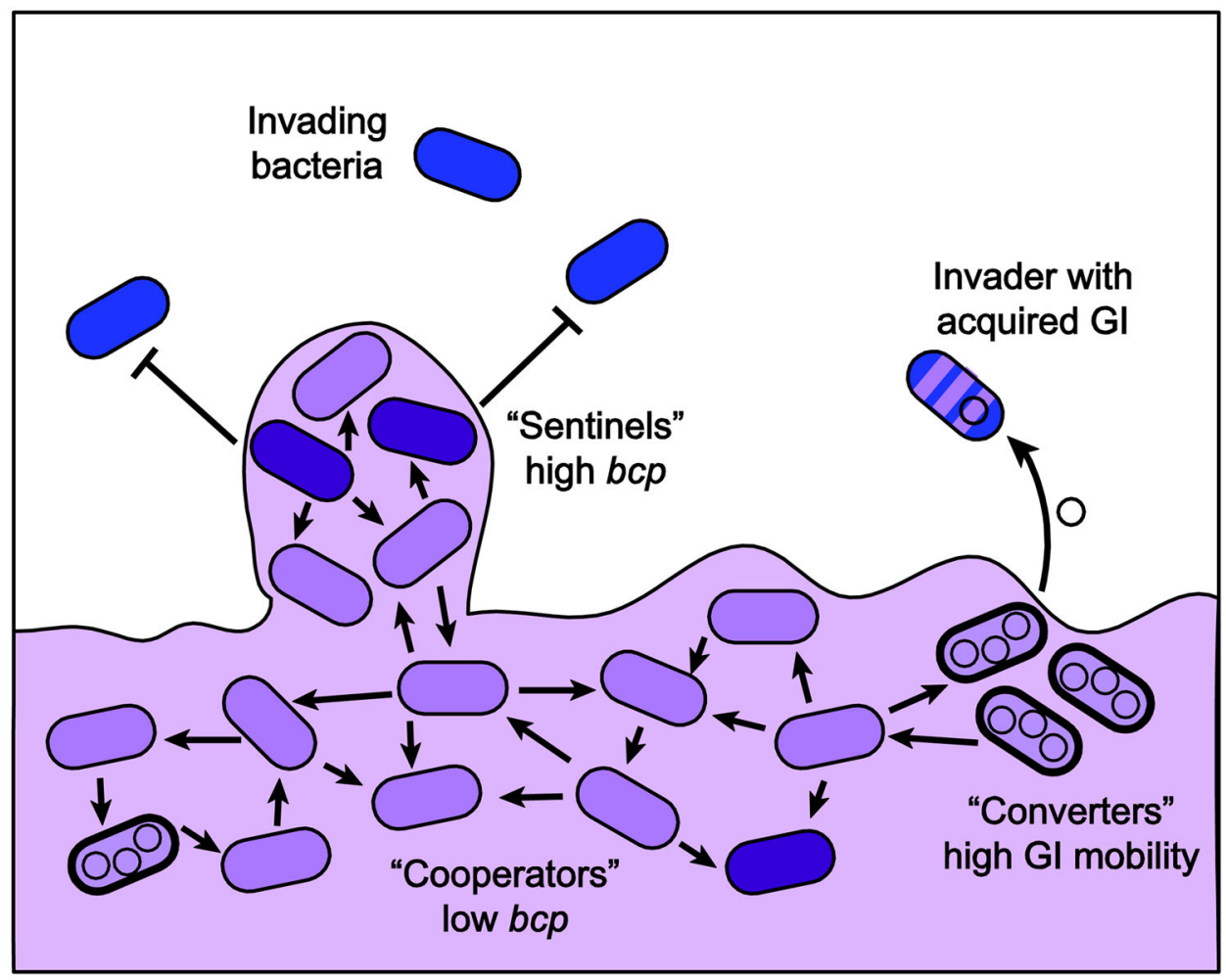

Figure 5 Key Figure. Model for CDI System-Mediated Competition and Cooperation in a Bacterial Community

The majority of Burkholderia thailandensis cells in a biofilm express bcpAIOB at a low level (light purple cells) and are predicted to cooperate via contact-dependent signaling (CDS). A small population of cells stochastically express $b c p A I O B$ at a high level (dark purple cells) and may function to exclude non-self bacteria (blue) from the community via contactdependent growth inhibition (CDI). Another subset of bacteria (circle-filled cells) may mobilize their $b c p A I O B$-containing genomic island (GI) and could transfer this element, potentially converting non-self invaders into self bacteria that would then express an identical $b c p A I O B$ allele. 